\title{
LINEAR PERTURBATIONS OF ORDINARY DIFFERENTIAL EQUATIONS ${ }^{1}$
}

\author{
AARON STRAUSS ${ }^{2}$ AND JAMES A. YORKE ${ }^{3}$
}

Abstract. We present several results dealing with the problem of the preservation of the stability of a system $x^{\prime}=A(t) x$ which is subject to linear perturbations $B(t) x$, or to perturbations dominated by linear ones.

1. We present several results dealing with the problem of the preservation of the stability and continuous dependence for a system $x^{\prime}=A(t) x$ to which is added a linear perturbation $B(t) x$, or a perturbation dominated by a linear one. In $\$ 2$ we show that certain linear perturbations satisfying $\int_{0}^{\infty}|B(t)| d t<\infty$, which have little effect on an exponentially stable linear system, have a rather significant effect on linear systems possessing a slightly weaker stability property. In $\S 3$ we consider $B(t) \equiv B$, a constant matrix, and we answer the following question: if $x=0$ is to be exponentially stable for $x^{\prime}=A(t) x+B x$ no matter which exponentially stable system $x^{\prime}=A(t) x$ is considered, then what special form must $B$ have? In $\$ 4$ we give a new proof and slight extension of the following known result [4]: a necessary and sufficient condition that a fundamental matrix of $x^{\prime}=A(t) x+R_{n}(t) x$ converge is that a fundamental matrix of $y^{\prime}=R_{n}(t) y$ converge as $n \rightarrow \infty$.

Definition. We say that a linear system

$$
x^{\prime}=A(t) x
$$

is exponentially stable if there exist constants $K \geqq 1$ and $\sigma>0$ such that

$$
\left|X(t) X^{-1}(s)\right| \leqq K e^{-\sigma(t-s)} \quad \text { for all } t \geqq s \geqq 0,
$$

where $X(t)$ denotes a fundamental matrix of (L).

Unless otherwise stated, all functions $f(t, x)$ considered here are required to be continuous for all $t \geqq 0$ and $x$ in $R^{d}, d \geqq 1$.

Received by the editors February 5, 1970.

AMS 1969 subject classifications. Primary 3451, 3453; Secondary 3440.

Key words and phrases. Stability, exponential stability, perturbations, continuous dependence, linear systems, convergence of solutions.

1 Sponsored by the Mathematics Research Center, United States Army, Madison, Wisconsin, under Contract No.: DA-31-124-ARO-D-462.

2 Supported in part by NSF grant GP-8914.

3 Supported in part by NSF grant GP-9347. 
2. The following result is known.

THEOREM 1. Let (L) be exponentially stable, with corresponding constants $K$ and $\sigma$. Let $g(t, x)$ satisfy any one of the following three conditions for all $t \geqq 0$ and $x$ in $R^{d}$.

$$
\begin{aligned}
& |g(t, x)| \leqq \gamma|x|, \text { where } \gamma<\sigma / K . \\
& |g(t, x)| \leqq \gamma(t)|x|, \text { where } t^{-1} \int_{0}^{t} \gamma(s) d s \rightarrow 0 \text { as } t \rightarrow \infty . \\
& |g(t, x)| \leqq \gamma(t), \text { where } \int_{t}^{t+1} \gamma(s) d s \rightarrow 0 \text { as } t \rightarrow \infty .
\end{aligned}
$$

Then all solutions of $x^{\prime}=A(t) x+g(t, x)$ approach zero as $t \rightarrow \infty$.

Stronger conclusions are also known, but this one suffices for our purpose. A proof using (2.1) may be found in [1], one using (2.2) in [3], and one using (2.3) in [5]. W. A. Coppel [2] has shown that the part using (2.1) is still true if one replaces the exponential stability of (L) by the weaker assumption that, for some $K \geqq 1$ and $\sigma>0$,

$$
\sup _{t \geqq 0} \int_{0}^{t}\left|X(t) X^{-1}(s)\right| d s \leqq K / \sigma .
$$

The purpose of this section is to show that the parts using (2.2) and (2.3) do not follow under the assumption (2.4), even when $\int_{0}^{\infty} \gamma(s) d s$ $<\infty$.

EXAMPLE 1. We construct a two-dimensional linear system (L) for which (2.4) holds, and we define a matrix $B(t)$ satisfying $\int_{0}^{\infty}|B(t)| d t$ $<\infty$, such that "most" solutions of $x^{\prime}=A(t) x+B(t) x$ are unbounded as $t \rightarrow \infty$. Define the intervals

$$
I_{n}=\left[n-2^{-(3 n+1)}, n+2^{-(3 n+1)}\right], \quad J_{n}=\left[n-2^{-3 n}, n+2^{-3 n}\right],
$$

for $n=1,2, \cdots$. Define two $C^{\infty}$ functions $\lambda(t)$ and $\phi(t)$ on $[0, \infty)$ such that

$$
\begin{aligned}
& \lambda(t)=2^{2 n} \quad \text { if } t \in I_{n}, \quad \phi(t)=2^{2 n} \quad \text { if } t \in I_{n}, \\
& =1 \quad \text { if } t \notin J_{n}, \quad=0 \quad \text { if } t \notin J_{n},
\end{aligned}
$$

and so that $\lambda(t)$ and $\phi(t)$ are each monotone in each component of $J_{n}-I_{n}, n=1,2, \cdots$. Thus

$$
\int_{J_{n}} \lambda(t) d t \leqq 2^{-n+1}, \quad \int_{J_{n}} \phi(t) d t \leqq 2^{-n+1}, \quad \int_{J_{n}} \phi(t) \lambda(t) d t \geqq 2^{n} .
$$


Now define

$$
A(t)=\left(\begin{array}{cc}
-1-\lambda^{\prime}(t) \lambda^{-1}(t) & 0 \\
0 & -\frac{1}{2}
\end{array}\right), \quad B(t)=\left(\begin{array}{cc}
0 & \phi(t) \\
0 & 0
\end{array}\right) .
$$

Therefore $\int_{0}^{\infty}|B(t)| d t<\infty$. We now show that (L) satisfies (2.4). A fundamental matrix is

$$
X(t)=\left(\begin{array}{cc}
\lambda^{-1}(t) e^{-t} & 0 \\
0 & e^{-t / 2}
\end{array}\right)
$$

Since

$$
\begin{aligned}
\int_{0}^{t} e^{-(t-s)} \lambda^{-1}(t) \lambda(s) d s & \leqq \int_{0}^{t} e^{-(t-s)} d s+\sum_{n=1}^{[t+1]} \int_{J_{n}} \lambda(s) d s \\
& \leqq 1+\sum_{n=1}^{\infty} 2^{-n+1}
\end{aligned}
$$

(2.4) holds for (L) with $K=6, \sigma=\frac{1}{2}$. However, there is a solution $y(t)$ $=\left(y_{1}(t), y_{2}(t)\right)$ of $y^{\prime}=A(t) y+B(t) y$ satisfying

$$
y_{1}(t)=\lambda^{-1}(t) e^{-t} \int_{0}^{t} e^{s} \lambda(s) \phi(s) e^{-s / 2} d s, \quad y_{2}(t)=e^{-t / 2}
$$

Hence

$$
\begin{aligned}
y_{1}\left(n+2^{-3 n}\right) & =e^{-n-2^{-3 n}} \int_{0}^{n+2^{-3 n}} e^{s / 2} \lambda(s) \phi(s) d s \\
& \geqq e^{-n-2^{-3 n}} e^{\left(n-2^{-3 n}\right) / 2} \int_{J_{n}} \lambda(s) \phi(s) d s \\
& \geqq\left(\frac{2}{e^{1 / 2}}\right)^{n} e^{-2^{-3 n}-2^{-3 n-1}} \rightarrow \infty \quad \text { as } n \rightarrow \infty
\end{aligned}
$$

EXAMPLE 2. We construct a one-dimensional linear system (L) for which (2.4) holds, and we define a function $\phi(t) \geqq 0$ satisfying $\int_{0}^{\infty} \phi(t) d t<\infty$, such that all solutions of $x^{\prime}=A(t) x+\phi(t)$ are unbounded as $t \rightarrow \infty$. Define $I_{n}, J_{n}, \lambda(t)$, and $\phi(t)$ as in Example 1. Then the linear (one-dimensional) system

$$
x^{\prime}=\left(-1-\lambda^{\prime}(t) \lambda^{-1}(t)\right) x
$$

satisfies (2.4). Also $\int_{0}^{\infty} \phi(t) d t<\infty$. But there is a solution $z(t)$ of

$$
z^{\prime}=\left(-1-\lambda^{\prime}(t) \lambda^{-1}(t)\right) z+\phi(t)
$$

satisfying 


$$
z(t)=\lambda^{-1}(t) e^{-t} \int_{0}^{t} e^{s} \lambda(s) \phi(s) d s \geqq y_{1}(t),
$$

where $y_{1}(t)$ is as in Example 1. Thus $z(t)$ is unbounded as $t \rightarrow \infty$. Since every solution of (2.5) is bounded as $t \rightarrow \infty$, it follows that all solutions of (2.6) are unbounded as $t \rightarrow \infty$.

Notice that in each of the above two examples, the perturbation is unbounded as $t \rightarrow \infty$. The problem of what happens if it is assumed to be also bounded remains open.

3. Let $a$ be the family of all $d \times d$ matrices $A(t)$, continuous on $[0, \infty)$, for which (L) is exponentially stable. We say that a matrix $B$ perturbs $a$ if $x^{\prime}=A(t) x+B x$ is exponentially stable for every $A(t) \in a$. This problem arises in the following way: suppose one knows that a system is both linear and exponentially stable. Suppose no further information about the system can be obtained. If this system is subject to linear perturbations $B x$, one would like to know that stability is preserved no matter what linear, exponentially stable unperturbed system (L) was at hand. This is the motivation behind asking that $B x$ perturb a whole class of equations (L). If $I$ denotes the identity matrix, then it is obvious that $\alpha I$ perturbs $a$ if and only if $\alpha \leqq 0$. It is perhaps surprising that even if $B$ is diagonal with all diagonal entries nonpositive, then $B$ does not perturb $a$ unless all the diagonal entries are equal. More generally, we have the following result.

Theorem 2. $B$ perturbs $a$ if and only if $B=\alpha I$ for some $\alpha \leqq 0$.

Proof. Let $B=\alpha I$ for some $\alpha \leqq 0$. Let $X(t)$ and $Y(t)$ denote fundamental matrices of (L) and

$$
y^{\prime}=A(t) y+B y,
$$

respectively, $X(0)=Y(0)=I$. Then $Y(t)=e^{\alpha t} X(t)$. Thus $B$ perturbs a.

Conversely, let $B \neq \alpha I$ for all $\alpha \leqq 0$. If $B=\alpha I$ for some $\alpha>0$, then $B$ clearly does not perturb $a$ (choose $A=-\frac{1}{2} B$ ). Thus suppose that $B \neq \alpha I$ for all real $\alpha$. Then $d \geqq 2$ and there exists $x_{1} \in R^{d}$ such that $x_{1}$ and $B x_{1}$ are linearly independent. Define $x_{2}=-x_{1}+B x_{1}$. Choose $x_{3}, \cdots, x_{d}$ so that $\left\{x_{1}, \cdots, x_{d}\right\}$ is a basis. Define $A$ such that $A x_{1}=-x_{1}-x_{2}, A x_{2}=x_{1}-x_{2}$, and $A x_{i}=-x_{i}$ for $i=3, \cdots, d$. Then $A \in q$ because

$$
e^{-t}\left(x_{1} \cos t+x_{2} \sin t\right), \quad e^{-t}\left(x_{1} \sin t-x_{2} \cos t\right), \quad x_{3} e^{-t}, \cdots, x_{d} e^{-t},
$$

form a set of $d$ linearly independent solutions to $x^{\prime}=A x$. But (3.1) 
is not exponentially stable because $A x_{1}+B x_{1}=0$. Thus $B$ does not perturb $Q$.

REMARK. In the "only if" part of the above proof, the matrix $A(t)$ is constant. Thus Theorem 2 holds with $Q$ replaced by $Q_{c}$, the family of all constant $d \times d$ matrices for which (L) is exponentially stable.

4. The purpose of this section is to give a new proof of the following result due to A. Ju. Levin [4], who proved the result in the case where $f(t, x)=A(t) x$.

THEOREM 3. Let $x_{n}(t), Y_{n}(t)$, and $x(t)$ satisfy

$$
\begin{aligned}
x^{\prime} & =f(t, x)+R_{n}(t) x, & x(0) & =x_{0}, \\
Y^{\prime} & =R_{n}(t) Y, & Y(0) & =I, \\
x^{\prime} & =f(t, x), & x(0) & =x_{0},
\end{aligned}
$$

respectively. Assume that solutions of (4.3) are uniquely determined by $x_{0}$. Let $T>0$. Then as $n \rightarrow \infty$

$$
Y_{n}(t) \rightarrow I \quad \text { uniformly on }[0, T]
$$

implies

$$
x_{n}(t) \rightarrow x(t) \text { uniformly on }[0, T] \text { for every } x_{0} \in R^{d} .
$$

If $f(t, x)=A(t) x$, then (4.5) implies (4.4).

Proof. Define $w_{n}(t)=Y_{n}^{-1}(t) x_{n}(t)$. Then $w_{n}(t)$ satisfies

$$
w^{\prime}=Y_{n}^{-1}(t) f\left(t, Y_{n}(t) w\right), \quad w(0)=x_{0} .
$$

Suppose $Y_{n}(t) \rightarrow I$ uniformly. Since the right-hand side of (4.6) converges to that of $(4.3), w_{n}(t) \rightarrow x(t)$ uniformly. Therefore $x_{n}(t) \rightarrow x(t)$ uniformly.

Now assume $f(t, x)=A(t) x$. Let $X_{n}(t)$ and $X(t)$ denote fundamental matrices of (4.1) and (4.3), respectively, satisfying $X_{n}(0)=X(0)=I$. Define $W_{n}(t)=Y_{n}^{-1}(t) X_{n}(t)$. Then $W_{n}(t)$ satisfies

$$
W^{\prime}=W X_{n}^{-1}(t) A(t) X_{n}(t), \quad W(0)=I .
$$

Suppose $X_{n}(t) \rightarrow X(t)$ uniformly. From (4.7) $W_{n}(t) \rightarrow W(t)$ uniformly, where $W(t)$ satisfies

$$
W^{\prime}=W X^{-1}(t) A(t) X(t), \quad W(0)=I .
$$

By inspection $X(t)$ satisfies (4.8). Hence $W(t)=X(t)$, i.e., $W_{n}(t)$ $\rightarrow X(t)$ uniformly. Therefore $Y_{n}(t) \rightarrow I$ uniformly. 
REMARKs. It is not known if (4.5) implies (4.4) in the nonlinear case. The implication " $(4.4) \Rightarrow(4.5)$ " seems to be more useful; nonetheless, it would be nice to be able to characterize (4.5) in terms of the simpler (4.4) in the nonlinear case. It would also be nice to characterize (4.4) in terms of $R_{n}(t)$. However, no such characterization is known. In particular Opial [6] has given an example showing that

$$
\left|\int_{0}^{t} R_{n}(s) d s\right| \rightarrow 0 \text { uniformly on }[0, T]
$$

does not imply (4.4). Of course (4.4) and (4.9) are equivalent if $R_{n}(t)$ is diagonal.

\section{REFERENCES}

1. E. A. Coddington and N. Levinson, Theory of ordinary differential equations, McGraw-Hill. New York, 1955. MR 16, 1022.

2. W. A. Coppel, Stability and asymptotic behavior of differential equations, D. C. Heath, Boston, Mass., 1965. MR 32 \#7875.

3. C. Corduneanu, Sur la stabilité asymptotique, An. Ști. Univ. "Al. I. Cuza" Iaşi Sect. I 5 (1959), 37-40. MR 22 \#3849a.

4. A. Ju. Levin, Passage to the limit for nonsingular systems $\dot{X}=A_{n}(t) X$, Dokl. Akad. Nauk SSSR 176 (1967), 774-777 = Soviet Math. Dokl. 8 (1967), 1194-1197. MR $37 \# 3140$.

5. A. Strauss and J. A. Yorke, Perturbation theorems for ordinary differential equations, J. Differential Equations 3 (1967), 15-30. MR 34 \#3029.

6. Z. Opial, Continuous parameter dependence in linear systems of differential equations, J. Differential Equations 3 (1967), 571-579. MR 35 \#6891.

University of Maryland, College Park, Maryland 20742

UNIVERSITY OF Wisconsin, MADISON, Wisconsin 53706 